\title{
Editorial
}

\section{Neurocardiology and Atherosclerosis: The Effect of Ethnic Differences on Heart-brain Interaction}

Recent developments in the fields of cardiology and neurology have led to the discovery that pathophysiologic entities once considered as solely having cardiovascular or neurological manifestations, may in fact affect both systems at the same time. Although the full extent of this heart-brain interaction is only now becoming apparent, there is substantial available evidence suggesting a multitude of connections between the two systems. The study of these interactions has become known as neurocardiology. ${ }^{[1]}$

The domain of neurocardiology is indeed a broad one. This is in part due to new discoveries in older established fields. For example, the physiological aspects of autonomic cardiac innervation have been studied extensively in the past. However, the recent revelations regarding shared pathophysiologic mechanisms between takotsubo cardiomyopathy and acute cardiac dysfunction secondary to subarachnoid hemorrhage, namely, both associated with massive catecholamine release, present new, and exciting insights as well as a completely new viewpoint on such syndromes. ${ }^{[2]}$ Another interesting example is the relationship between heart failure and cognitive decline, recently documented by Havakuk et al. Both functional and anatomic brain abnormalities were detected in heart failure patients in the aforementioned study, with some being associated with neurohormonal, nutritional, or inflammatory mechanisms. ${ }^{[3]}$ The fact that atrial fibrillation may result in cognitive dysfunction before overt transient ischemic attacks or stroke is of additional interest. ${ }^{[1]}$ Finally, cardiac surgery may be followed by cognitive dysfunction that may not always be associated with cardiopulmonary bypass

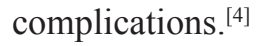

Atherosclerosis does not constitute an exception as far as heart-brain interaction is concerned. Admittedly, our understanding of the pathophysiology and time course of atherosclerosis has increased dramatically. A significant role is currently attributed to intimal inflammation and local immune responses, endothelial dysfunction, disturbed laminar flow in blood vessels, and alterations in endothelial mechanotransduction as causative mechanisms. ${ }^{[5]}$ A number of associated risk factors are also currently known, including but not limited to smoking, hypercholesterolemia, diabetes mellitus, hypertension, disturbed lipid homeostasis and increased high-sensitivity C-reactive protein. Strikingly, even though the entirety of the circulatory system is exposed to the same risk factors, the distribution of atherosclerotic lesions is not uniform. Notably, the internal mammary artery is known to be highly resistant to atherosclerosis. More importantly, this nonuniformity is not only prevalent within the same circulatory system but also within different ethnic groups. For instance, Asian populations appear to have a higher risk of intracranial atherosclerosis compared to Caucasians while ethnic minorities residing in the United States have been found to have differences in risk factors compared to their nonemigrant compatriots. ${ }^{[6,7]}$ These suggest both genetic as well as environmental influences at hand.

Srinivasan et al. reported a cross-sectional study on postcoronary artery bypass graft (CABG) surgery patients in this issue of the Journal of Neurosciences in Rural Practice. ${ }^{[8]}$ In this study, 210 patients of Asian descent who underwent CABG between 1998 and 1999 were examined for neck vessel and cerebrovascular disease using neck vessel Doppler ultrasonography, brain magnetic resonance imaging (MRI) with T1, T2, T2-flair, and magnetic resonance angiography (MRA) sequences as well as head computed tomography (CT) scanning. Data on traditional risk factors were also collected for this patient group. Dyslipidemia as well as combined diabetes mellitus with hypertension was the most commonly occurring risk factors $(57 \%$ and $37 \%$, respectively). An additional $20 \%$ of patients had no identifiable risk factor while only $4 \%$ were smokers. Forty-one percent of patients had right-sided and $35 \%$ had left-sided weakness. Neck vessel Doppler ultrasonography was not pathologic in $59 \%$ of patients, with only $7.2 \%$ of the rest having significant identifiable disease. Ninety-one percent of all patients exhibited varying degrees of cerebrovascular disease be it symptomatic or not based on the detection of hyperintense T2 lesions on MRI examination, despite the large proportion of nonpathologic neck Doppler scans. MRA sequences, in contrast, identified $30 \%$ of patients with normal neck vessels and $33.8 \%$ of patients with combined common and the left/right carotid artery involvement. The authors conclude that in this population, medium- and small-sized intracranial vessels are most commonly affected, with large vessel involvement being rare ( $82 \%$ and $23.8 \%$ of patients with detected abnormalities, respectively). This is in contrast to Caucasian populations where extracranial carotid disease is most prevalent.

These findings are very relevant not only for the field of neurocardiology but also for everyday clinical practice 
as the treatment of large-vessel cerebrovascular disease differs significantly from that of medium/small-vessel disease. In addition, they prompt the adoption of a more critical outlook for health-care practitioners involved in the treatment of non-Caucasians. In this study, neck Doppler ultrasonography clearly underestimated lesions in comparison to MRA, due in part to the fact that MRA is more sensitive for intracranial regions that are apparently more affected in Asian populations. Thus, MRA might be considered earlier in this patient group. Finally, these findings contribute to a greater body of evidence linking heart-brain interactions, not necessarily explained by traditional risk factors and thus creating new research opportunities.

\section{George Markousis-Mavrogenis}

Onassis Cardiac Surgery Center, Athens, Greece

Address for correspondence: Mr. George Markousis-Mavrogenis, Onassis Cardiac Surgery Center, 50 Esperou Street, 17561 Athens, Greece.

E-mail: georgemm32@gmail.com

\section{REFERENCES}

1. van der Wall EE. The brain-heart connection; a round trip. Neth Heart J 2011;19:269-70.

2. Kurisu S, Kihara Y. Interaction between brain and heart. Circ J 2016;80:1905-6.

3. Havakuk O, King KS, Grazette L, Yoon AJ, Fong M, Bregman $\mathrm{N}$, et al. Heart failure-induced brain injury.
J Am Coll Cardiol 2017;69:1609-16.

4. Bruggemans EF. Cognitive dysfunction after cardiac surgery: Pathophysiological mechanisms and preventive strategies. Neth Heart J 2013;21:70-3.

5. Tabas I, García-Cardeña G, Owens GK. Recent insights into the cellular biology of atherosclerosis. J Cell Biol 2015;209:13-22.

6. Pu Y, Dou X, Liu L. Natural history of intracranial atherosclerotic disease. Front Neurol 2014;5:125.

7. Kuller LH. Ethnic differences in atherosclerosis, cardiovascular disease and lipid metabolism. Curr Opin Lipidol 2004;15:109-13.

8. Srinivasan K, Ravikumar S, Chandra SR, Ganapathy S, Ravi GS Cerebral and coronary vasculature in disease associations and dissociations in the South Indian Population. J Neurosci Rural Pract 2017;8:352-6.

This is an open access article distributed under the terms of the Creative Commons Attribution-NonCommercial-ShareAlike 3.0 License, which allows others to remix, tweak, and build upon the work non-commercially, as long as the author is credited and the new creations are licensed under the identical terms.

\begin{tabular}{|l|l|}
\hline \multicolumn{2}{|c|}{ Access this article online } \\
\hline Quick Response Code: & Website: \\
\hline & \\
\cline { 2 - 2 } & \\
\hline
\end{tabular}

How to cite this article: Markousis-Mavrogenis G. Neurocardiology and atherosclerosis: The effect of ethnic differences on heart-brain interaction. J Neurosci Rural Pract 2017;8:504-5. 